\title{
The Application of Pentaprism Scanning Technology on the
}

\author{
Manufacturing of M3MP \\ Erhui Qi ${ }^{* a}$, Haixiang $\mathrm{Hu}^{\mathrm{a}}$, Haifei Hu${ }^{\mathrm{a}, \mathrm{b}}$, Glen Cole ${ }^{\mathrm{c}}$, Xiao Luo ${ }^{\mathrm{a}}$, Virginia Ford ${ }^{\mathrm{c}}$, \\ Xuejun Zhang ${ }^{\mathrm{a}}$ \\ ${ }^{a}$ Changchun Institute of Optics, Fine Mechanics and Physics, Chinese Academy of \\ Sciences, Changchun 130033, Jilin, China; \\ ${ }^{b}$ University of Chinese Academy of Sciences, Beijing 100049, China \\ ${ }^{\mathrm{c}}$ Thirty Meter Telescope, Pasadena, CA 91105, USA
}

\begin{abstract}
The PSS (pentaprism scanning system) has advantages of simple structure, needless of reference flat, be able of on-site testing, etc, it plays an important role in large flat reflective mirror's manufacturing, especially the high accuracy testing of low order aberrations. The PSS system measures directly the slope information of the tested flat surface. Aimed at the unique requirement of M3MP, which is the prototype mirror of the tertiary mirror in TMT (Thirty Meter Telescope) project, this paper analyzed the slope distribution of low order aberrations, power and astigmatism, which is very important in the manufacturing process of M3MP. Then the sample route lines of PSS are reorganized and new data process algorism is implemented. All these work is done to improve PSS's measure sensitivity of power and astigmatism, for guiding the manufacturing process of M3MP.
\end{abstract}

Keywords: Optical testing, pentaprism, scanning test, flat mirror, slope measurement

\section{INTRODUCTION}

$\mathrm{TMT}^{[1]}$ (the Thirty Meter Telecope) is one of the several ultra-huge telescopes human beings constructing, its tertiary mirror (M3M) is a huge elliptical flat mirror, with a size of $2.5 \mathrm{~m} \times 3.5 \mathrm{~m}$. The $\mathrm{M} 3 \mathrm{M}$ will be the largest flat mirror in the world, and its main final manufacturing requirements is low order aberrations (Plate Scale) below 18.8 mas, single subaperture's root mean square of slope values (SlopeRMS) below $0.8 \mu \mathrm{rad}$. A prototype of M3M (M3MP) is now being manufactured by the Key Laboratory of Optical System Advanced Manufacturing Technology (KLOMT) in Changchun Institute of Optics, Fine Mechanics and Physics (CIOMP), which has the same requirements of M3M.

For testing large aperture flat mirror like M3M, traditional methods like Ritchey-Common and Fizeau interferometer tests are not practical: Ritchey-Common test needs a similar standard sphere and the testing optical is hard to construct; limited by the size of reference flat, the interferometer testing needs many apertures to stitching, Sub-aperture stitching will lose the testing accuracy of low order aberrations especially power and astigmatism, which M3M cares much.

*E-mail: 13578916792@163.com, Tel: +8643186708687

AOMATT 2016: Large Mirrors and Telescopes, edited by Myung Kyu Cho, Bin Fan, Proc. of SPIE Vol. 9682 , $96821 \mathrm{~A} \cdot$ ㅇ 2016 SPIE · CCC code: $0277-786 \mathrm{X} / 16 / \$ 18 \cdot$ doi: 10.1117/12.2245813 
Different with the interferometer tests, the Pentaprism scanning technology is an absolute testing methods. Pentaprisms have unique of deflecting light beams at a constant angle (nominally by $90^{\circ}$ ) regardless of its orientation in the line of sight direction, using this advantage, beams will scans over the mirror and gets the surface profile by measuring slopes. The PPS testing use the mirror itself as the reference, so another reference is not needed.

\section{BASIC PRINCIPLE}

As shown in figure 1, a pentaprism can deviate the incident beam perfectly 90 degree to the test surface regardless of the orientation in the pitch direction. Thus the result measurements are independent of prism alignment, for this reason, the pentaprism can be used to scanning the test surface.
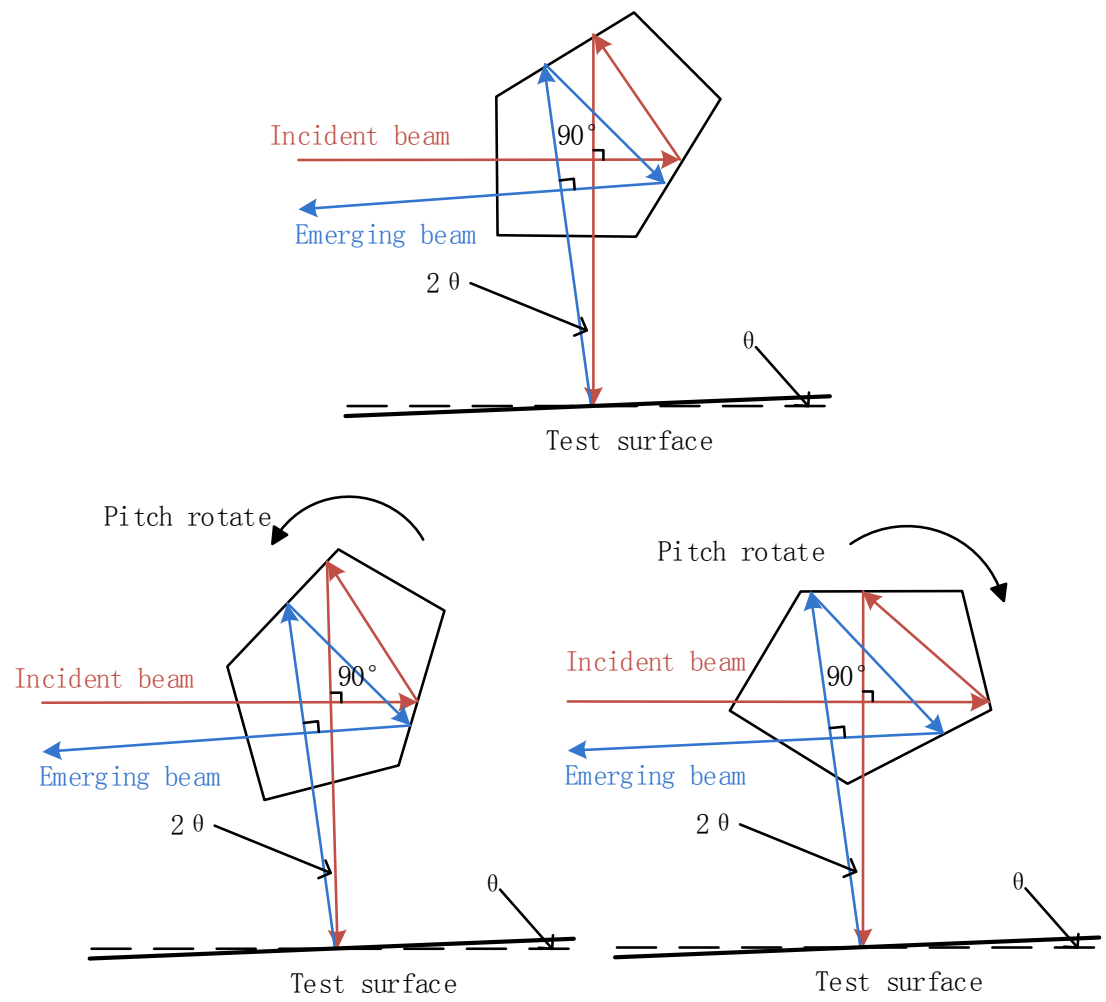

Figure 1 Principle of a pentaprism

The principle of pentaprism scanning system (PPS) is shown in figure 2, two pentaprisms (one is called the reference prism, A , and the other is the scanning prism, B ) co-aligned to a high accuracy autocollimator (autocollimator1 in figure 2), the measuring beam comes out from the autocollimator, then deviated by the pentatprim to the mirror surface, the reflected beam would take the surface information back. The autocollimator can measure one single signal once time, so two 
electronic-controlled shutter are designed for alternatively selecting the reference and the scanning signal. The difference between the reference and the scanning represent the surface slope varieties, as shown in equation (1):

$$
\theta=\frac{1}{2}\left(\mathrm{~V}_{\text {scan }}-\mathrm{V}_{\text {ref }}\right)
$$

in which Vscan represent the slope information back from the scanning prim, and Vref represent the slope information back from the reference prim. Using this referencing measurement, some common errors for both prisms such as autocollimator's rotation, tested mirror's tilt will be avoided. By performing different lines' scanning, the whole surface's profile can be measured.

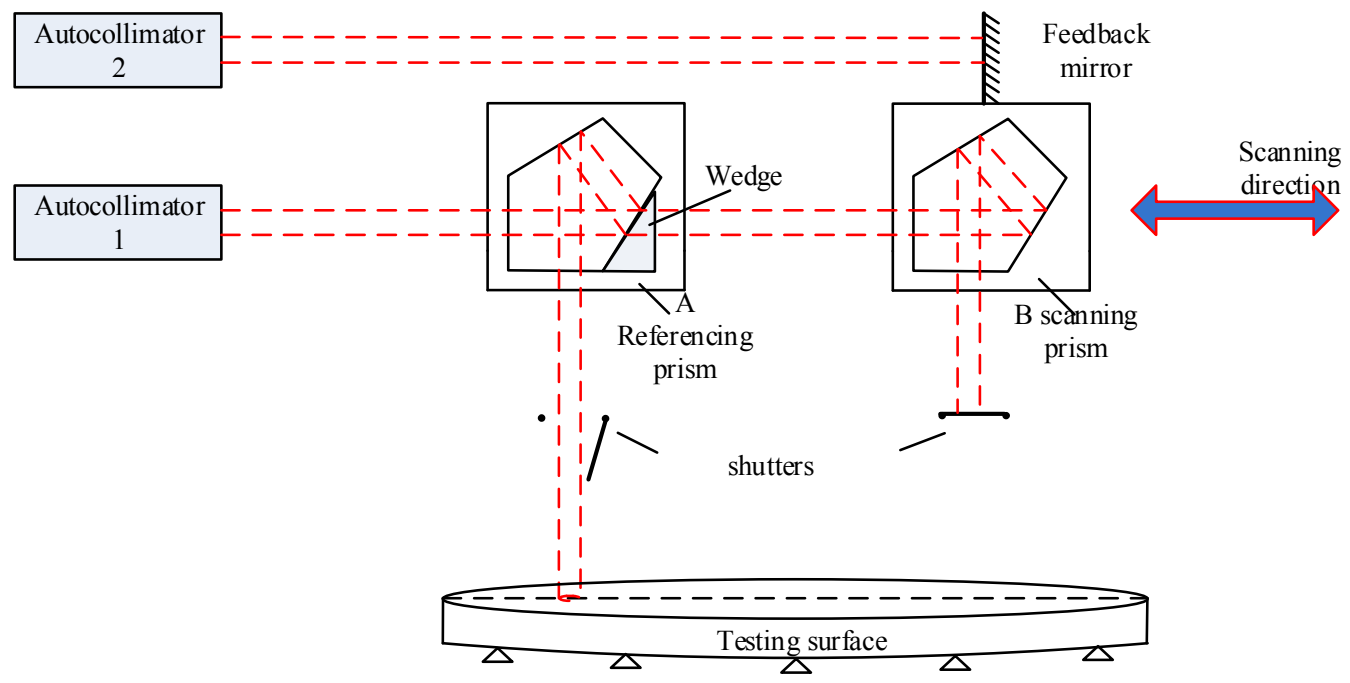

Figure 2 Principle of the pentaprism scanning system

While performing scanning lines, angular motion of the scanning pentaprim will introduce additional errors to the measuring point, so another autocollimator (autocollimator2 in figure 2) is used to construct a feed-back control of the angular motions of the scanning pentaprism.

\section{PLATE SCALE ANALYSIS}

M3MP has a special requirement of low order aberrations, the Plate Scale, which defined by power and astigmatism. In optical manufacturing, Zernike polynomials are usually used to express optical aberrations, power and astigmatism are expressed by equation (2):

$$
\begin{aligned}
& Z_{4}=2 \rho^{2}-1 ; \\
& Z_{5}=\rho^{2} \cos (2 \theta) ; \\
& Z_{6}=\rho^{2} \sin (2 \theta) ;
\end{aligned}
$$

in which Z4 is power, Z5 is 0 degree astigmatism and Z6 is 45 degree astigmatism. 
Only considering the low order aberrations which M3MP cares much, then Plate Scale can be described by equation (3), which is a linearity of power and astigmatism:

$$
Z=a^{*} Z_{4}+b^{*} Z_{5}+c^{*} Z_{6}
$$

in equation(3), a, b, c respectively represents the coefficient of Z4, Z5 and Z6, changing into polar coordinates, equation (3) can be described by:

$$
Z=a^{*}\left(2 * \rho^{2}-1\right)+b^{*}\left(\rho^{2} * \cos (2 \theta)\right)+c^{*}\left(\rho^{2} * \sin (2 \theta)\right)
$$

Taking partial derivation of radius $\rho$, we can get,

$$
Z_{\rho}=4 * a * \rho+2 * b * \rho * \cos (2 \theta)+2 * c * \rho * \sin (2 \theta)
$$

There is only one order radius $\rho$ left except the coefficients, taking two-order partial derivation of radius $\rho$, then we can get,

$$
Z_{\rho \rho}=4 * a+2 * b * \cos (2 \theta)+2 * c * \sin (2 \theta)
$$

After taking two-order partial derivation of radius $\rho$, we can see that the Plate Scale is a function of radian, $\theta$. Take a combination of the trigonometric functions, we can get

$$
Z_{\rho \rho}=4 * a+2 * B * \cos (2(\theta-\varphi))
$$

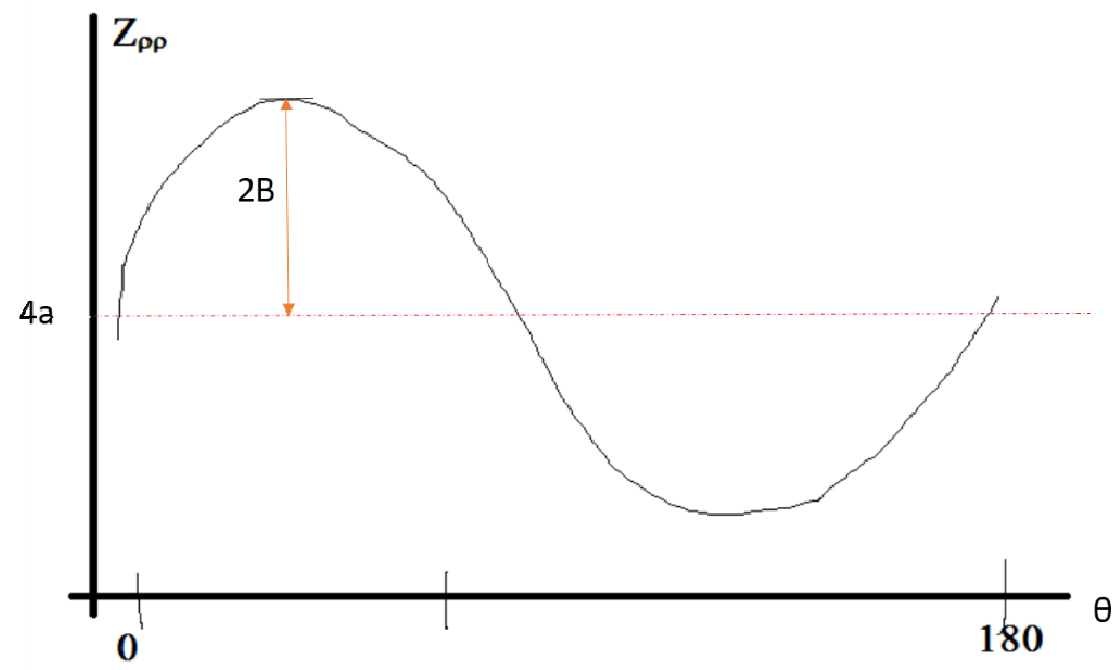

Figure 3 Form of Plate Scale's two-order partial derivation of radius $\rho$

As shown in figure 3, after taking two-order partial derivation of $\rho$, the Plate Scale can be described by a cosine function, the coefficient of $Z 4$ can be represented by the cosine function's global offset, the coefficient of Z5 and Z6 can be represented by the cosine function's amplitude and phase offset.

Proc. of SPIE Vol. 9682 96821A-4 


\section{TESTING ON M3MP}

A special pentaprism scanning system is designed for M3MP, the system is designed to bridged the manufacturing rotational table, which provides an on-site testing. It is also kinematic system which can be lifted and removed when not in use.as shown in figure 4.

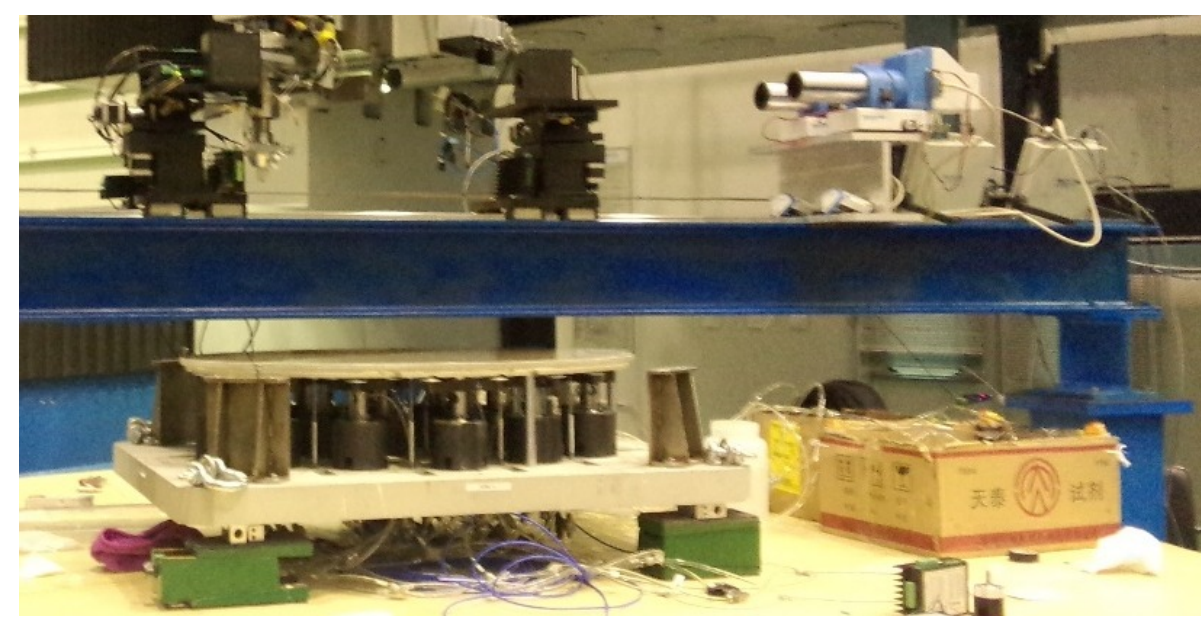

Figure 4 PPS measuring on M3MP

In the testing on M3MP, we performed 6 lines scanning to fully describe the cosine function shown by the equation (7), and the corresponding radians are respectively degree $0,30,60,90,120$, 150. By performing the 6 lines' scanning, the data from these lines are fitted to the cosine function shown in equation (7), then the coefficients of power and astigmatism can be achieved.

The low order aberrations from the PPS and the mid\&high frequency information from sub-aperture stitching result are combined, to be the surface input for the next polishing circle. 


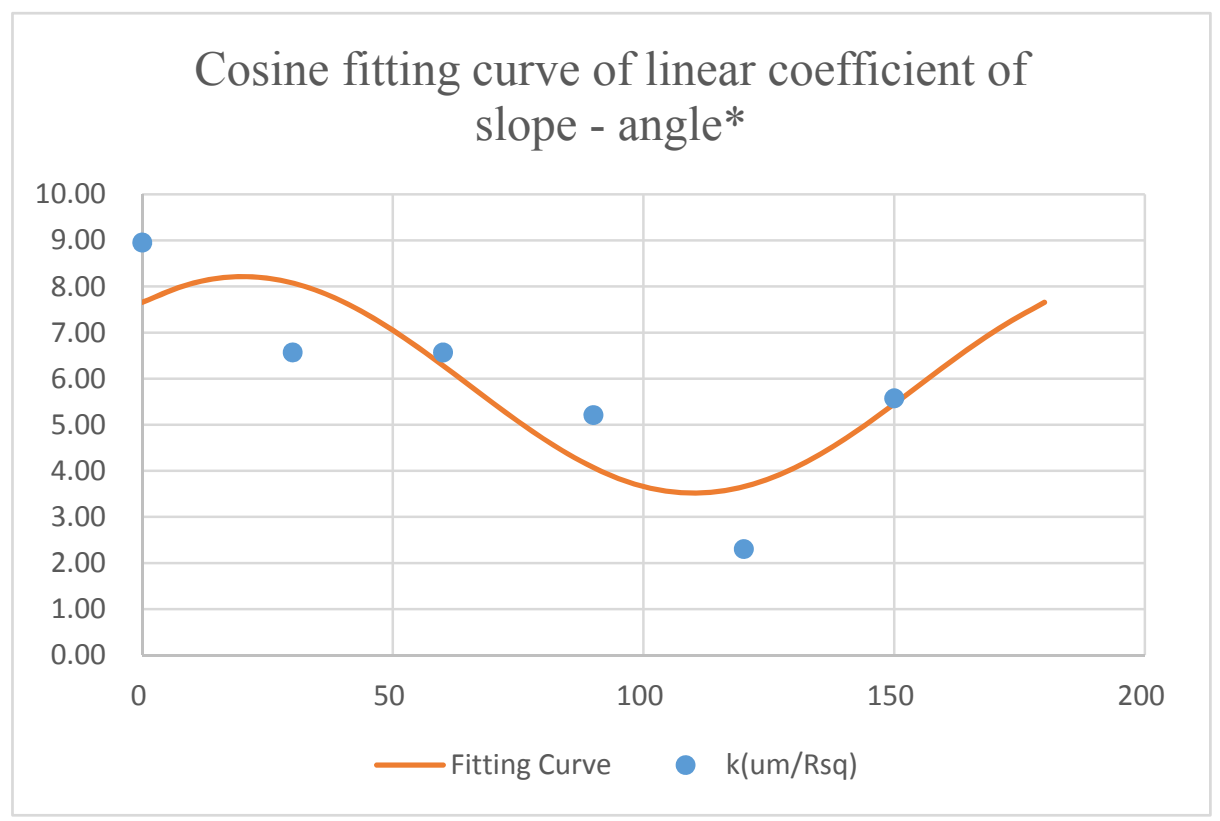

Figure 5 Measurement points and the fitted curve for single testing

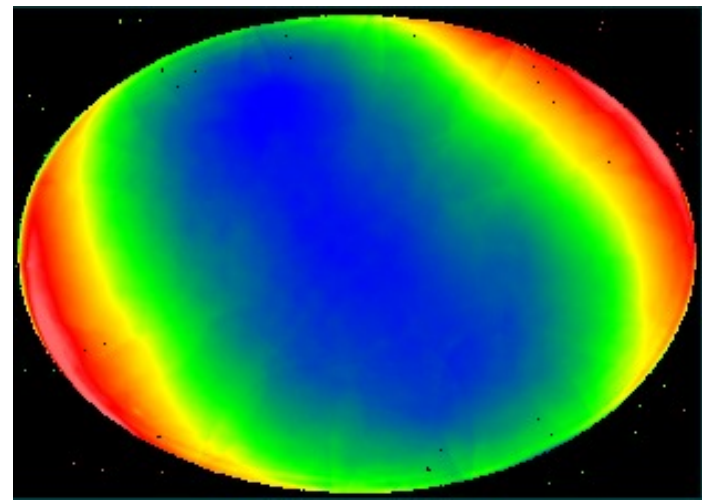

Before $($ Plate scale $=21.67)$

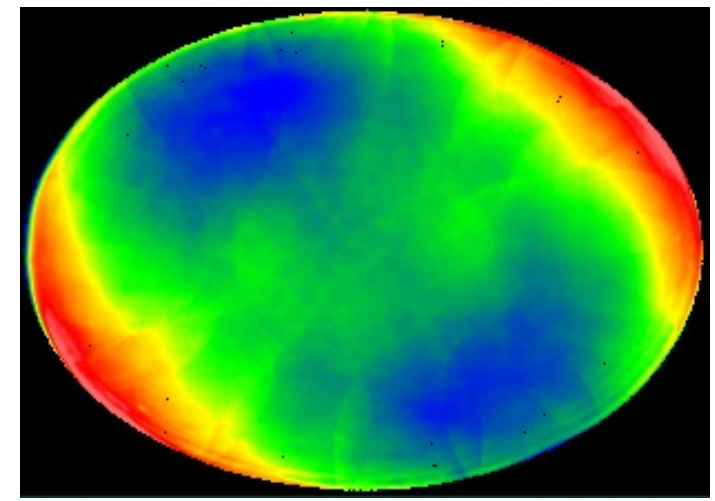

After (Plate scale=7.73)

Figure 6 Surface changes before and after one polishing circle

Figure 5 shows the measured points and the fitted curve for single testing, figure 6 shows the Surface changes before and after one polishing circle, after one polishing recycle, the Plate Scale, which M3MP cares much, decreases from 21.67 to 7.73 , that's a satisfying result.

\section{CONCLUSIONS AND FUTURE WORK}

M3MP has a special requirement of low order aberrations, defined as Plate Scale. After analyzing Plate Scale, we found a new way to perform the data process for pentatprism scanning, which shows significative guidance for the manufacture of M3MP.

We also found that if there is much high frequency errors on the mirror, the fitting residual errors will increase, we will concentrate on studying high frequency errors' influence in the future. 


\section{ACKNOWLEDGEMENT}

We gratefully thank Virginia Ford, Glen Cole for their helpful advices about the

manufacturing of M3MP. This work is financially supported by Natural Science Foundation of China No.61210015 and Chinese National 973 program No.2011CB013205.

\section{REFERENCES}

[1] TMT Group. Tertiary Mirror Surface Figure Specification [R]. TMT.OPT.SPE.12.001.DRF02, 2012.

[2] Sen Han, Erik Novak, Mike Schurig. Ritchey-Common Test used for Measurement of Astronomical Optical[J]. Proc. SPIE, 2003, 4842: 271-273.

[3] C. Zhao, R. Sprowl, M. Bray, J. H. Burge. Figure Measurements of a Large Optical Flat With a Fizeau Interferometer and Stitching Technique[J]. Proc. SPIE,2006, 6293: 62930K.

[4] Julius Eldon Yellowhair. Advanced technologies for fabrication and testing of large flat mirrors[D]. Arizona: the University of Arizona(College of Optical Sciences), 2007.

[5] P. Mallik, C. Zhao, J. H. Burge. Measurement of a 2-m flat using a pentaprism scanning system[J]. Proc. SPIE, 2005, 5869: 58691A.

[6] J. Yellowhair, J. H. Burge. Analysis of a scanning pentaprism system for measurement of large flat mirrors [J].Applied Optics, 2007,46(35):8466-8474

[7] R. J. Noll, “Zernike polynomials and atmospheric turbulence,” J. Opt. Soc. Am. 66, 207-211 (1976).

[8] V. N. Mahajan, "Zernike polynomials and wavefront fitting," in Optical Shop Testing, 3rd ed., D.Malacara, ed.(Wiley, 2007) pp. 498-546.

[9] Qi Erhui, Luo Xiao, Zheng Ligong etal. Optical testing of large flat mirror with non-circular pupil based on scanning pentaprism technology[J]. Journal of Optoelectronics•Laser.2014,7(25):1370-1375.(in Chinese) 